\section{Bronchoconstriction following nebulised colistin in cystic fibrosis}

\author{
S Cunningham, A Prasad, L Collyer, S Carr, I Balfour Lynn, C Wallis
}

\begin{abstract}
Nebulised colistin is regularly used as antipseudomonal therapy in children with cystic fibrosis. We assessed bronchoconstriction in response to nebulised colistin in 58 children. Nebulised colistin significantly reduced $\mathrm{FEV}_{1}, \mathrm{MEF}_{25 \%}$, and $\mathrm{SaO}_{2}$ for 15 minutes. In 20 children the reduction was greater than $10 \%$ from baseline $\mathrm{FEV}_{1}$, and was still at that level in five at $30 \mathrm{~min}$ utes. Subjective assessment, baseline $\mathrm{FEV}_{1}$, and serum IgE were unable to identify susceptible children. It is recommended that children receiving colistin should be carefully assessed for bronchoconstriction.

(Arch Dis Child 2001;84:432-433)
\end{abstract}

Keywords: cystic fibrosis; colistin; bronchoconstriction

Nebulised antibiotic therapy is considered to have significant impact on long term pulmonary morbidity in patients with cystic fibrosis (CF) colonised with Pseudomonas aeruginosa. ${ }^{1}$ Although a number of antibiotics can be nebulised, colistin is a popular choice in European centres, primarily because of the Danish evidence supporting its use. Colistin sulphomethate is a polymyxin antibiotic that is preservative free; it is produced as a dry powder preparation which is reconstituted with saline prior to nebulisation.

Studies in adults have shown bronchoconstriction in response to nebulised colistin ${ }^{2}$; however, to date no study has identified the extent of the problem in children. We therefore sought to identify the frequency of bronchoconstriction following nebulised colistin, its duration of effect, and its relation to subjective feelings of chest tightness.

NHS Trust,

Whitechapel, London

E1 1BB, UK

L Collyer

S Carr

Royal Brompton \&

Harefield NHS Trust,

Sydney Street, London

SW3 6NP, UK

I Balfour Lynn

Correspondence to:

Dr Cunningham

steve.cunningham@talk21.com

Accepted 16 November 2000 Methods

The study was a prospective cross sectional assessment of children attending three large paediatric CF clinics in London, UK. Children ( $>5$ years of age) who were regular or first time users of nebulised colistin were asked to participate in the study, and were asked not to use any bronchodilator for at least six hours prior to the study. Spirometry was performed immediately before nebulisation of a standard dose of colistin made up to a total of $4 \mathrm{ml}$ vol- ume with $0.9 \%$ saline ( $<7$ years 0.5 megaunit, $\geqslant 7$ years 1 megaunit). Spirometry was performed using a compact spirometer (Vitalograph, Buckingham, UK). Colistin was administered by either Pari LC star (Pari, Starnberg, Germany) or Ventstream (Medic-Aid, Pagham, Sussex, UK) nebulisers and either Portaneb (Medic-Aid, Pagham, Sussex, UK) or Pariboy (Pari, Starnberg, Germany) compressors. Further spirometry was performed immediately following the cessation of nebulisation and then at 15 minutes post nebulisation. Children with bronchoconstriction at 15 minutes had further spirometry performed at 30 minutes. Polgar standards have been used where age/sex related values of spirometry are quoted. ${ }^{3}$ Following nebulisation, children were asked to give a subjective appraisal of whether or not they felt chest discomfort in association with the treatment.

Descriptive statistics report mean (standard deviation) unless otherwise stated. Percentage changes are relative to baseline, and not a percentage fall of predicted. Student's $t$ tests were used to assess change in spirometry before and after nebulisation and also changes in relation to subjective discomfort. Spearman correlation was used to test the association between age and recent serum IgE value with changes in spirometry.

Ethical approval was obtained from the local ethical committee at each of the three hospitals participating. Informed consent was obtained from parents and children prior to each study.

\section{Results}

A total of 58 children were studied with a mean age of 12.0 (3.1) years (range 5.1-17.4). Fifty five children were regular colistin users (41 for more than two years); three were using colistin for the first time. Mean $\mathrm{FEV}_{1}$ before colistin was $59.3 \%(18.3 \%)$.

Colistin was associated with a significant fall $5.9 \%(9.6 \%), \mathrm{p}<0.001$ ) and 15 (mean $3.3 \%$ $(10.2 \%), \mathrm{p}<0.004)$ minutes post nebulisation. Twenty children (34\%) had more than $10 \%$ reduction in $\mathrm{FEV}_{1}$; these children had a mean decrease in $\mathrm{FEV}_{1}$ of $17.8 \%$ (5.9\%), with a maximum fall of $37.5 \%$. The time to maximum fall in $\mathrm{FEV}_{1}$ was 0 minutes in 13, 15 minutes in five, and 30 minutes in two patients. Five children (9\%) continued to have an $\mathrm{FEV}_{1}$ 


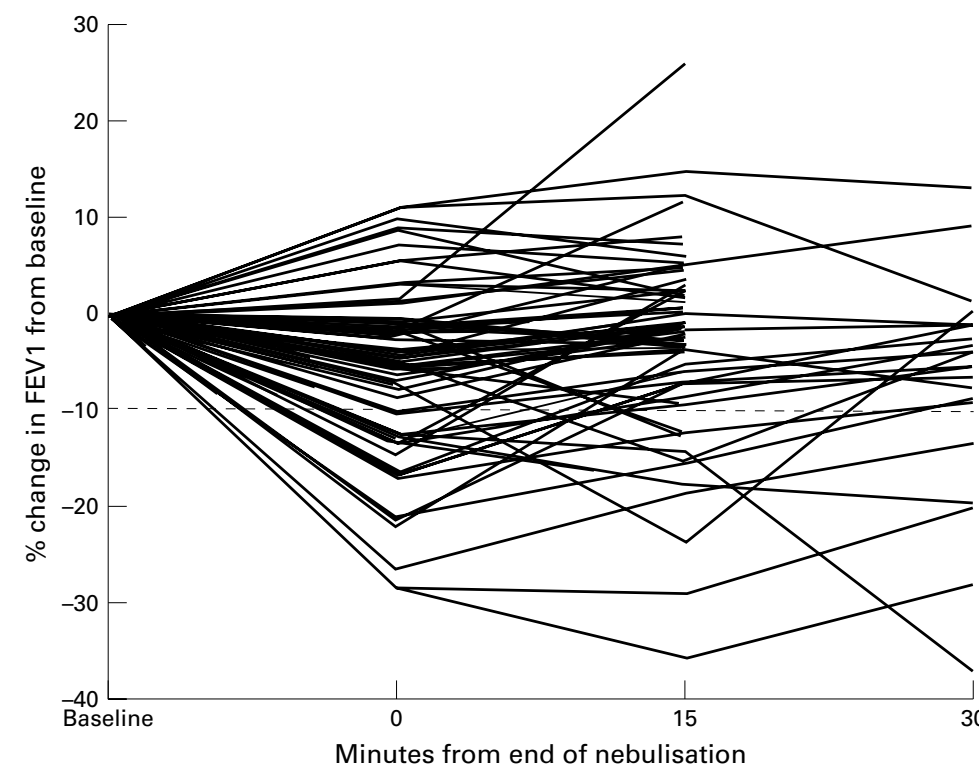

Figure 1 Change in $F E V_{1}$ from baseline with nebulised colistin.

at $10 \%$ above baseline at 30 minutes (see fig 1). In the group as a whole there was also a significant reduction in $\mathrm{MEF}_{25 \%}$ at 0 (mean reduction $12.2 \%(35.0 \%), \mathrm{p}=0.003)$ and 15 (mean $10.5 \%(37.6 \%), \mathrm{p}<0.001)$ minutes, and a reduction in oxygen saturation at 0 (mean $0.6 \%(1.3 \%), \mathrm{p}<0.001)$ and 15 (mean $0.4 \%$ $(1.2 \%) \mathrm{p}=0.023)$ minutes. Sixteen children $(28 \%)$ had a reduction in oxygen saturation of at least $2 \%$ following nebulised colistin (maximum reduction $4 \%$ ).

No significant correlation was found between age, serum IgE, or baseline $\mathrm{FEV}_{1}$ and changes in $\mathrm{FEV}_{1}, \mathrm{MEF}_{25 \%}$, or $\mathrm{SaO}_{2}$ at 0,15 , and 30 minutes. No significant difference was found for changes in spirometry post nebulisation between those patients experiencing chest discomfort in association with nebulisation ( $\mathrm{n}=9, \mathrm{p}=0.15)$, or those with current symptoms of respiratory tract infection $(n=10$, $\mathrm{p}=0.85)$.

\section{Discussion}

This study has shown significant bronchoconstriction in one third of children receiving nebulised colistin in our clinics. This bronchoconstriction was persistent (30 minutes) in $9 \%$, and was associated with a fall in oxygen saturation in some children. Atopic tendency, baseline $\mathrm{FEV}_{1}$, and subjective assessment were not able to identify those children with significant bronchoconstriction.

Nebulised colistin has previously been reported as inducing bronchoconstriction in adult patients ${ }^{2}$ : this effect was independent of the tonicity of the solution nebulised. Two studies have assessed bronchoconstriction in response to nebulisation of the intravenous preparation of tobramycin in groups of $12^{4}$ and $26^{5} \mathrm{CF}$ patients. The latter study showed bronchoconstriction in 19\% of patients immediately following nebulisation; however, they did not address time to minimum $\mathrm{FEV}_{1}$ or time to recovery. A new preparation of tobramycin (TOBI, PathoGenesis, Seattle, USA) has been reported as having no significant bronchoconstriction; however, patients were only studied at 30 minutes and some bronchoconstriction was evident at that time, though it is unclear in how many patients. ${ }^{6}$ The cost of this new tobramycin preparation, however, will possibly limit its use in Europe and colistin will most likely remain the first choice nebulised antibiotic in the paediatric age group. It is important therefore that users are aware of the potential bronchoconstriction that may be induced by this antibiotic. Children should be tested for bronchoconstriction with first administration of colistin; in younger children who are unable to perform spirometry, the use of bronchodilator premedication is recommended.

We acknowledge Adriana Machin, senior physiotherapist, Royal Brompton \& Harefield NHS Trust, and Gail Slade and John Martin, respiratory technicians, Great Ormond Street Hospital.

1 Mukhopadhyay S, Singh M, Cater JI, et al. Nebulised antipseudomonal antibiotic therapy in cystic fibrosis: a meta analysis of benefits and risks. Thorax 1996;51:364-8.

2 Dodd ME, Abbott J, Maddison J, et al. Effect of tonicity of nebulised colistin on chest tightness in adults with cystic fibrosis. Thorax 1997;52:656-8.

3 Polgar G, Weng T. The functional development of the respiratory system from the period of gestation to adulthood. Am Rev Respir Dis 1979;120:625-95.

4 Chua HL, Collis GG, Le-Souef PN. Bronchial response to nebulised antibiotics in children with cystic fibrosis. Eur Respir f 1990;3:1114-16.

5 Ramogopal M, Lands LC. Inhaled tobramycin and bronchial hyperactivity in cystic fibrosis. Pediatr Pulmonol 2000;29:366-70.

6 Ramsey BW, Pepe MS, Quan JM, et al for the Cystic Fibrosis Inhaled Tobramycin Study Group. Intermittent administration of inhaled tobramycin in patients with cystic fibrosis. N Engl f Med 1999;340:23-30. 